\title{
La voluntad anticipada, tutores y directrices avanzadas: caso de simulación clínica
}

\author{
Decision-making capacity, surrogates, and advance \\ directives: a clinical simulation case \\ Daniel Enrique Rodríguez-Bauzá,* Miguel Enrique Silva-Rodríguez,* David Rodgers*
}

\begin{abstract}
Palabras clave:
Autonomía del paciente, cuidados al final de la vida, ética, directivas anticipadas, sustitutos, caso de simulación.

Keywords:

Patient autonomy, end of life care, ethics, advance directives, surrogates, simulation case.
\end{abstract}

\section{RESUMEN}

El principio ético de autonomía refleja la autodeterminación que los pacientes deben tener para tomar decisiones en cuestiones de salud. En este caso de simulación de una hora de duración, paciente "Y", de 75 años de edad, sexo masculino, profesor de escuela primaria retirado, con antecedentes de salud neoplasia de pulmón, es llevado al Departamento de Emergencias por su hija debido a un historial de una semana con empeoramiento de tos y dolor torácico (punta de costado derecha). Durante las últimas 24 horas, confundido, toma del estado general y distrés respiratorio. El paciente firmó documento de voluntad anticipada o "directiva avanzada" hace tres meses, el cual específica "no intubación”. La simulación transcurre durante la ronda de la mañana durante la cual el estado del paciente continúa deteriorándose y éste requiere intubación. Su acompañante (hija con ocho meses de embarazo) expresa su voluntad de que su padre pueda vivir lo suficiente para ver el nacimiento de su primer nieto, ella no conoce acerca de la existencia de este documento legal. Los objetivos de esta actividad permiten el desarrollo de habilidades comunicativas y un curso de acción para resolver conflictos éticos en toma de decisiones de salud en un entorno de aprendizaje simulado seguro. Este caso de simulación clínica es una herramienta educativa experiencial que puede mejorar la competencia profesional, la confianza y la colaboración de los estudiantes en el desempeño de la práctica profesional en concordancia con el principio ético de autonomía y autodeterminación del paciente.
The ethical principle of autonomy reflect the selfdetermination of patients to make decisions in health matters. In this 1-hour simulation case, patient " $Y$ ", 75-year-old male, retired elementary school teacher, with a history of lung cancer, is taken to the emergency department by his daughter due to one week history of worsening cough and pleuritic chest pain. During the last 24 hours, he has become more confused, lethargic, fatigue, and worsening respiratory distress. The patient signed an advance directive 3 months ago, which specifies "no intubation". The simulation takes place in the morning round during which the patient's condition continues to deteriorate and requires intubation. Her companion (daughter with eight months of pregnancy) expresses her wish that "her father can live long enough to see the birth of his first grandchild", she does not know about the existence of this legal document. The objectives of this simulation case allow the development of communication skills and the best course of action to resolve this ethical conflict in a safe simulated learning environment. This clinical simulation case is an experiential educational tool that can improve professional competence, confidence and collaboration of students in the performance of professional practice in accordance with the ethical principle of autonomy and self-determination of the patient.

\section{INTRODUCCIÓN}

$\mathrm{L}$ a tradición hipocrática que vinculaba valores $ـ$ profesionales basados en la responsabilidad y beneficencia del médico para con sus pacientes fue progresivamente siendo sustituida por la nueva bioética, en que se destaca la importancia de la autonomía del paciente como consumidor de servicios médicos y actor principal, y se sustituye el enfoque tradicional de la dignidad de toda vida humana por una preocupación referida a la calidad de la vida de los pacientes de corte utilitarista y pragmático. ${ }^{1}$ A menudo existen conflictos entre médicos, enfermeras, otros miembros del equipo de atención médica, pacientes y miembros de la familia sobre cuánto y qué 
tipo de atención tiene sentido para alguien con una esperanza de vida limitada. La planificación anticipada es un proceso que ayuda a los adultos de cualquier edad o etapa de salud a establecer sus decisiones sobre la atención médica que desean recibir durante enfermedades graves y crónicas, ${ }^{2}$ garantizando que las personas reciban una atención médica que sea coherente con sus valores morales, objetivos y preferencias. En el año 1990, la Corte Suprema de los Estados Unidos de Norteamérica especificó que las personas tienen el derecho constitucional de aceptar o rechazar cualquier tipo de tratamiento médico o quirúrgico indicado; este hecho fue el resultado del Acta de Autodeterminación del Paciente de 1991. ${ }^{3-6}$ La autonomía como valor fundamental de la medicina norteamericana ha contribuido al surgimiento del concepto de directivas avanzadas, ${ }^{6}$ término que comprende un instrumento legal llamado "Directiva de los Médicos en el Acta de Muerte Natural de los Estados Unidos y la Carta Poder para la Atención Médica". Por ejemplo, una directiva brinda instrucciones al personal médico con respecto a la alimentación por sonda, el uso de ventiladores mecánicos o la suspensión de resucitación cardiopulmonar.

El marco jurídico de la voluntad anticipada en Latinoamérica es desigual; por ejemplo, múltiples leyes, decretos y órdenes autonómicas que se han expedido en España para regular la voluntad anticipada; por otro lado, en México las legislaciones especiales vigentes a nivel local son realmente pocas, lo cual provoca un vacío legal que se traduce en múltiples conflictos jurídicos. ${ }^{7}$ En el Cuadragésimo Quinto Periodo Ordinario de Sesiones de la Asamblea General de la Organización de los Estados Americanos (OEA) se adoptó la Convención Interamericana sobre la Protección de los Derechos Humanos de las Personas Mayores (A-70) regula la obligación de los estados parte de implementar un proceso para que la persona mayor pueda manifestar, de manera expresa, su voluntad anticipada e instrucciones respecto de las intervenciones en atención de la salud. Chile, Argentina, Ecuador y El Salvador han ratificado este instrumento. ${ }^{8}$ La ética médica es un elemento obligatorio de la formación formal de los médicos estadounidenses. ${ }^{9}$ La familiaridad con los principios éticos en un nivel básico es necesaria para aprobar los exámenes de licencia médica en Estados Unidos. Sin embargo, muchos médicos son incapaces de dilucidar principios éticos específicos, explicar cómo o por qué han surgido los principios de la ética médica, o integrar y priorizar sistemáticamente los principios de la ética médica. ${ }^{10} \mathrm{De}$ forma concomitante, sería importante incorporar una evaluación estandarizada de la enseñanza de la educación ética, por un motivo más relacionado con la teoría educativa: "no se puede enseñar ética si no demuestras que tu programa es efectivo"; del mismo modo que los programas de formación clínica deben demostrar su efectividad, los de formación ética también. ${ }^{11}$ La simulación permite tanto el entrenamiento como la evaluación que se pueden estandarizar y no presenta ninguno de los riesgos para el paciente asociados con el aprendizaje experiencial realizado en el entorno clínico real. ${ }^{12}$ Esta actividad educativa se divide en pre-debriefing (5 minutos), escenario de simulación 15 minutos y debriefing (40 minutos). El caso se presenta en su totalidad para los facilitadores e incluye las acciones críticas que los participantes deben realizar; lo cual se puede utilizar como referencia durante el debriefing. El facilitador puede utilizar el escenario preprogramado, el cual fue realizado en SimDesigner de Laerdal (previamente probado) o ajustar el maniquí (SimMan 3G) manualmente. También se incluye una herramienta para el debriefing y un cuestionario para evaluar la actividad después de la sesión de debriefing. El objetivo de este caso de simulación es fomentar la preparación ética de los participantes (ej. médicos, enfermeros, internos, residentes, estudiantes de enfermería y medicina) en la temática de la voluntad anticipada, tutores y directivas avanzadas; en un ambiente educativo seguro. Esperamos que esta herramienta educativa impacte positivamente la prestación de los servicios de salud.

Los objetivos específicos son:

1. Demostrar una evaluación adecuada de la capacidad para la toma de decisiones médicas.

2. Practicar habilidades comunicativas para resolver conflictos éticos.

3. Ilustrar el mejor curso de acción durante una situación ética al final de la vida.

4. Establecer las responsabilidades interprofesionales en la implementación de protocolos para la obtención de documentos de voluntad anticipada o directivas avanzadas en pacientes hospitalizados y en la atención primaria de salud.

5. Relacionar el código de ética médica (nacional o centro de trabajo) con acciones y/o decisiones tomadas durante la simulación. 


\section{PRESENTACIÓN DEL CASO}

Paciente, sexo masculino, 75 años de edad, profesor de escuela primaria retirado, es llevado al Departamento de Emergencias por su hija debido a un historial de empeoramiento de una semana con tos y dolor torácico (punta de costado derecha). Durante las últimas 24 horas, más confusión, toma del estado general y distrés respiratorio. El paciente tiene antecedentes de salud de neoplasia de pulmón, en cuarto ciclo de combinación quimioterapia con cisplatin y etoposide de un total de seis. Ingresado por diagnóstico de neumonía hace un mes, la cual se trató con tratamiento antibiótico intravenosos. De acuerdo con su hija, su tos y fatiga nunca cedieron, y hace tres días tubo fiebre de $39.5^{\circ} \mathrm{C}$ (termómetro axilar).

Resumen del examen físico:

1. Estado general: agotado, en distrés respiratorio.

2. Signos vitales: en el monitor de cabecera.

3. Aparato respiratorio: abovedamiento discreto del hemitórax derecho. Disminución de la expansión torácica. Vibraciones vocales abolidas en el hemitórax derecho. Matidez a la percusión y resistencia al dedo que percute en el hemitórax derecho. Hemitórax izquierdo normal.

Exámenes complementarios:

(i) Informe radiológico en el Departamento de Emergencias (radiografía de tórax posteroanterior [PA] y lateral): opacidad de límites poco precisos en el hemitórax derecho de aspecto horizontal; que borra los ángulos costofrénicos y cardiofrénicos. Desplazamiento del mediastino hacia el hemitórax izquierdo, espacios intercostales distendidos y hemidiafragma derecho descendido. Impresión radiológica: cáncer de pulmón metastásico con derrame pleural maligno.

Se realizó en horas de la mañana toracocentesis terapéutica, extrayendo $1 \mathrm{~L}$ de fluido pleural que fue enviado al laboratorio clínico para análisis. Además, se inició terapia antibiótica de combinación con ceftriaxona ( 1 g IV diario) más un macrólido: azitromicina (500 mg IV diario).

(ii) Tomografía computarizada de tórax de alta resolución: diagnóstico radiológico de neumonía postobstructiva que afecta el parénquima del lóbulo inferior derecho.
El paciente es admitido en el Servicio de Medicina Interna en horas de la noche, con diagnóstico al ingreso: cáncer de pulmón metastásico complicado con derrame pleural maligno y neumonía postobstructiva.

El paciente tiene en su historia médica un documento de voluntad anticipada o "directivos avanzados", el cual especifica "no intubación". Su hija, que tiene ocho meses de embarazo acompaña al paciente, ella no conoce acerca de la existencia de este documento legal. Durante su primer día de estadía hospitalaria, el estado del paciente se deteriora y la acompañante expresa su voluntad de que su padre pueda vivir lo suficiente para ver el nacimiento de su primer nieto, diciendo: Siento que esto es lo que él hubiera querido. A pesar del tratamiento proporcionado, el estado del paciente continúa deteriorándose y éste requiere intubación.

Implementación:

1. Lugar: Servicio de Medicina Interna (u otro servicio hospitalario dependiendo del rol de los participantes).

2. Ubicación temporal-espacial: ronda de la mañana.

3. Paciente: maniquí adulto (SimMan 3G) en cama de hospital con monitores de cabecera, bomba de infusión con solución salina $0.9 \%$ y mascarilla de alta concentración. Riñonera con esputo sanguinolento (esto simula hemoptisis).

4. Personal: dos actrices en los roles de enfermera confederada e hija de paciente, esta última con ocho meses de embarazo. Los participantes en la simulación mantienen su papel de proveedores médicos. El instructor puede estar presente físicamente para guiar a los participantes a través del escenario de simulación o detrás de escena, según el tipo de estudiantes y/o experiencia en simulación.

Uno de los participantes asumirá el rol de jefe de residentes y dirigirá el pase de visita o ronda de la mañana. Inicialmente se les debe dar tiempo a los alumnos (1 a 5 minutos) para familiarizarse con el caso e introducirse al acompañante. Durante este periodo inicial el paciente se encuentra estable.

Información en el monitor y ajustes al maniquí (0-5 minutos en la simulación):

1. $\mathrm{O}_{2}$ Sat: $97 \%$ con mascarilla de alta concentración $(8 \mathrm{~L} / \mathrm{min})$. 
2. Electrocardiograma: taquicardia sinusal.

3. Frecuencia respiratoria: 24 respiraciones por minuto.

4. Frecuencia cardiaca: 107 latidos por minuto.

5. Tensión arterial: $140 / 88 \mathrm{mmHg}$.

6. Temperatura: $37.8^{\circ} \mathrm{C}$.

7. Sonido vocal: gemidos y tos.

El estado clínico del paciente cambia después de 5 minutos, éste se caracteriza por una disnea angustiosa y progresiva, y cianosis (signos vitales debajo) $y$, a pesar del tratamiento actual y/o acciones tomadas por el equipo médico presente (ej. respiración de rescate con suplemento de oxígeno en el "Ambu Bag"), el paciente tiene una desaturación más pronunciada y distrés respiratorio.

Información en el monitor y ajustes al maniquí (5 minutos en la simulación):

1. Saturación de $\mathrm{O}_{2}: 80 \%$.

2. Frecuencia respiratoria: 7 respiraciones por minuto.

3. Frecuencia cardiaca: 130 latidos por minuto.

4. Tensión arterial: 146/90 mmHg.

Esta situación clínica mérita la intubación del paciente. Los participantes deben realizar una evaluación completa de la capacidad del paciente en cuestión, para la toma de decisiones (examen físico completo y la obtención de un historial apropiado).

El acompañante del paciente (hija) se comunica con los participantes:

1. Emociones: expresión facial de preocupación y ansiedad.

2. Diálogo:

Hija de paciente (ocho meses de embarazo):

- Haga todo lo posible para salvar su vida.

- Quiero que mi padre viva lo suficiente para ver el nacimiento de su primer nieto. Eso es lo que él hubiera querido.

Cuando los participantes verbalizan la necesidad de intubar al paciente, la enfermera confederada les informa que el paciente firmó un documento de voluntad anticipada o directivas avanzadas en el que se declara explícitamente "no intubación".

Aunque consideramos que la relación médico-paciente es una interacción dinámica en la cual el médico se guía por la comprensión, las preferencias y el comportamiento del paciente ${ }^{12}$ entre otros factores, el uso del protocolo SPIKES ${ }^{13}$

SPIKES es una sigla en inglés, cada letra representa una fase en este esquema de 6 pasos $^{13}$
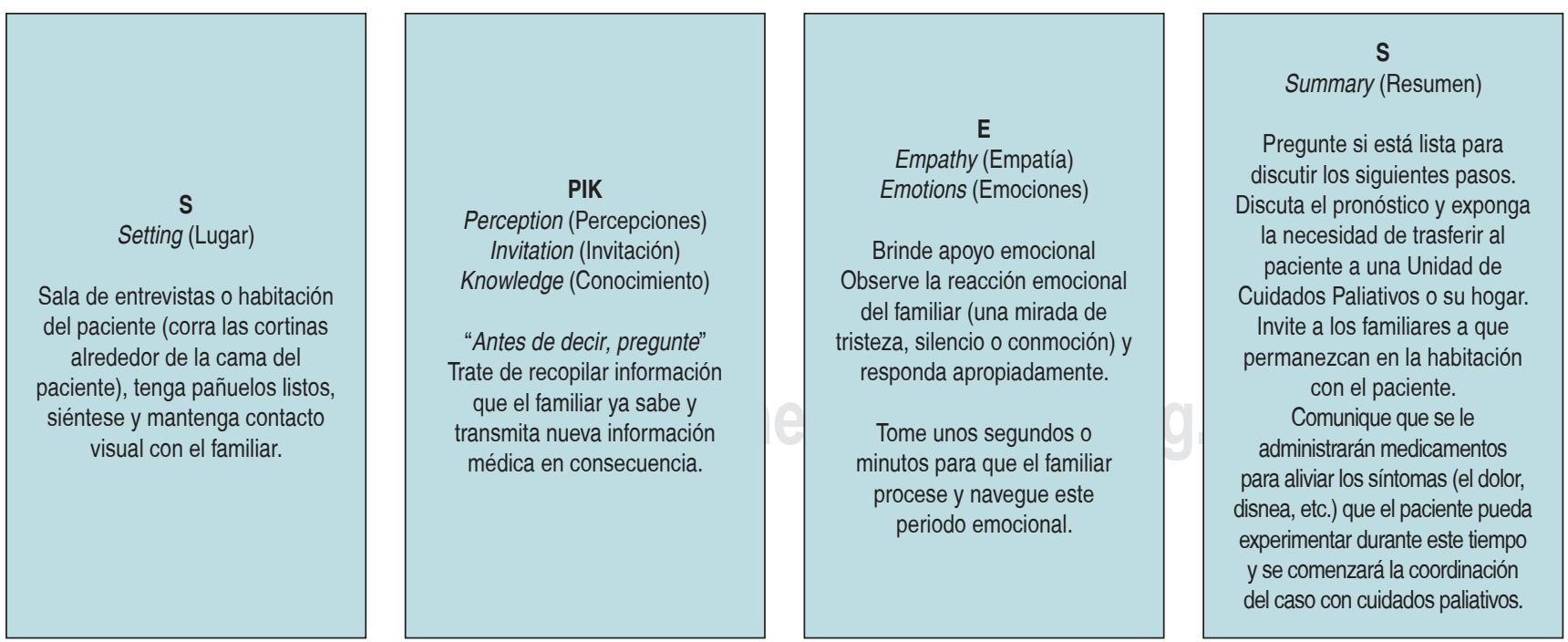

Figura 1: Protocolo SPIKES para comunicarse con el familiar. 


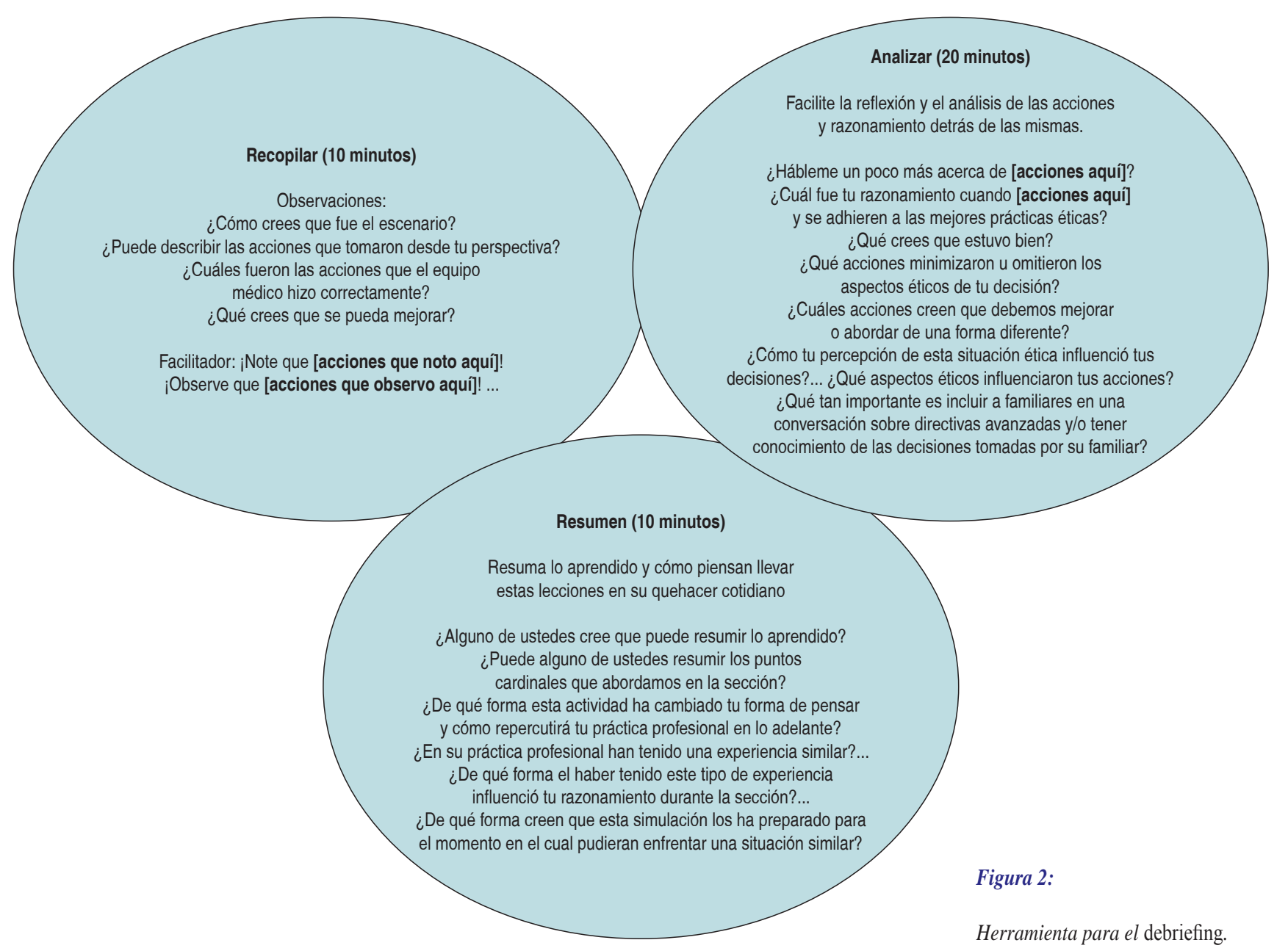

(siglas en inglés) posibilita una comunicación efectiva y organizada para que el equipo médico informe al familiar de manera oportuna, que el documento de voluntad anticipada o directivas avanzadas documenta claramente el deseo del paciente de no ser intubado y tiene prioridad sobre los deseos de los familiares del paciente (Figura 1).

\section{DISCUSIÓN}

El debriefing posterior a la simulación es uno de los componentes más eficaces de la educación basada en simulación y la piedra angular de la experiencia de aprendizaje en el entorno de la simulación médica. Éste permite a los profesores y alumnos reexaminar la experiencia del caso simulado, compartir su modelo mental y fomentar el razonamiento detrás de su juicio clínico. ${ }^{14} \mathrm{En}$ vez de un interrogatorio unidireccional, recomen- damos que el facilitador cree un espacio seguro en el cual los participantes puedan compartir opiniones y discutan sus ideas y puntos de vista abiertamente creando una excelente oportunidad para reflexionar y formular ideas concretas, así como trazar modos de actuación acorde con los estándares éticos. El amplio desarrollo en el campo de la simulación clínica a nivel mundial ha posibilitado la creación de múltiples herramientas para el debriefing. Por su sencillez recomendamos el uso del modelo de tres pasos Gather-AnalyzeSummarize (GAS), ${ }^{15}$ el cual hemos traducido al español con las siglas RAR (recopilar, analizar y resumir) en la Figura 2.

\section{CONCLUSIONES}

El envejecimiento poblacional está ocurriendo a un ritmo sin precedentes y se acelerará en las próximas décadas según data de la Organización 
Mundial de la Salud, especialmente en los países en desarrollo. ${ }^{16}$ Una encuesta nacional sobre cuidados a largo plazo en adultos mayores en Estados Unidos estimó que los cuidadores en el último año de vida fueron cónyuges, hijos y otros (ej. amigos). ${ }^{17}$ Los avances tecnológicos en la medicina moderna han cambiado las normas de muerte natural. $Y$ aunque hemos avanzado tecnológicamente, muchas de las opciones terapéuticas actuales no prometen la recuperación, éstas pueden mantener la vida con o sin una existencia significativa o con apoyo secundario (como sondas de alimentación, ventiladores, etc.). Por ello la práctica médica moderna debe replantear cómo validamos el principio ético de autonomía. Una "directiva anticipada y/o documento de voluntad anticipada" permite a las personas competentes diseñar y documentar su plan de decisiones de atención médica con anticipación en caso de una futura discapacidad o enfermedad terminal. A pesar de que la mayoría de los pacientes han oído hablar de las directivas anticipadas, pocos toman la iniciativa de redactar una directiva anticipada. La enseñanza de este principio ético se puede lograr con una combinación de aprendizaje didáctico y experiencial (ej. casos de simulación). La simulación presentada cuenta con todas las herramientas para ser una experiencia educativa exitosa, sola o en combinación con otros recursos publicados. Es una premisa en el futuro, evaluar el impacto de esta actividad en los modos de actuación de los profesionales y directamente en los servicios de salud.

\section{AGRADECIMIENTOS}

Agradecemos el apoyo del Centro de Simulación Clínica del Centro Médico Penn State Health Milton S. Hershey por promover programas de formación con simulación para mejorar la seguridad y la atención del paciente en Latinoamérica.

\section{REFERENCIAS}

1. Rodríguez YE. Temas para una bioética latinoamericana. Acta Bioeth. 2009; 15 (1): 87-93.

2. Sudore RL, Lum HD, You JJ, Hanson LC, Meier DE, Pantilat SZ, et al. Defining advance care planning for adults: a consensus definition from a multidisciplinary delphi panel. J Pain Symptom Manage. 2017; 53 (5): 821-832.e1. doi: 10.1016/j. jpainsymman.2016.12.331.

3. Johnson SH. After Cruzan. The U. S. Supreme Court's decision settles the case but raises new questions. Health Prog. 1990; 71 (8): 38-41, 57.
4. Kelley K. The patient self-determination act. a matter of life and death. Physician Assist. 1995; 19 (3): 49, 53-56, 59-60 passim.

5. Koch KA. Patient self-determination act. J Fla Med Assoc. 1992; 79 (4): 240-243.

6. Torchia DM. Advance directives. Physician Assist. 1992; 16 (5): 79-80, 83-84, 87.

7. Scholten G, Bourguignon S, Delanote A, Vermeulen B, Van Boxem G, Schoenmakers B. Advance directive: does the GP know and address what the patient wants? Advance directive in primary care. BMC Med Ethics. 2018; 19 (1): 58. doi: 10.1186/s12910-018-0305-2.

8. Sánchez Barroso JA. La voluntad anticipada en España y en México: Un análisis de derecho comparado en torno a su concepto, definición y contenido. Bol Mex Der Comp. 2011; 44 (131): 701-734. Disponible en: http://www.scielo.org.mx/scielo.php?script =sci arttext\&pid $=$ S0041-86332011000200008\&lng $=\overline{\text { es }}$ \&tlng $=\mathrm{es}$

9. Organización de los Estados Americanos. Convención Interamericana sobre la Protección de los Derechos Humanos de las Personas Mayores (A-70). Washington, D.C., Estados Unidos: 2015. [Acceso 05 de enero de 2021] Disponible en: http://www.oas.org/es/sla/ ddi/tratados_multilaterales_interamericanos_A-70_ derechos_humanos_personas_mayores_firmas. asp\#Argentina

10. Giubilini A, Milnes S, Savulescu J. The medical ethics curriculum in medical schools: present and future. J Clin Ethics. 2016; 27 (2): 129-145.

11. Young M, Wagner A. Medical ethics. In: StatPearls [Internet]. Treasure Island (FL): StatPearls Publishing; 2021. Available in: https://www.ncbi.nlm.nih.gov/ books/NBK535361/

12. Esquerda M, Pifarré J, Roig H, Busquets E, Yuguero $\mathrm{O}$, Viñas J. Evaluando la enseñanza de la bioética: formando «médicos virtuosos» o solamente médicos con habilidades éticas prácticas. Aten Primaria. 2019; 51 (2): 99-104. doi: 10.1016/j.aprim.2017.05.018.

13. Buckman R. Breaking bad news: a guide for health care professionals. Baltimore: Johns Hopkins University Press; 1992. p. 15.

14. Khalili H. Clinical simulation practise framework. Clin Teach. 2015; 12 (1): 32-36.

15. Abulebda K, Auerbach M, Limaiem F. Debriefing techniques utilized in medical simulation. In: StatPearls [Internet]. Treasure Island (FL): StatPearls Publishing; 2021.

16. Phrampus $\mathrm{P}, \mathrm{O}^{\prime}$ Donnell J. Debriefing using a structured and supported approach. In: The comprehensive textbook of healthcare simulation. New York: Springer; 2014. pp. 73-85.

17. Cai X, Robinson J, Muehlschlegel S, White DB, Holloway RG, Sheth KN et al. Patient preferences and surrogate decision making in neuroscience Intensive Care Units. Neurocrit Care. 2015; 23 (1): 131-141. doi: 10.1007/s12028-015-0149-2.

\section{Correspondencia:}

Daniel Enrique Rodríguez Bauzá.

500 University Drive, Hershey, PA 17033.

Teléfono: +1 (717) 531 -4099.

E-mail:drodriguezbauza@icloud.com 